Pacific Journal of Mathematics

COMPLETE HOLOMORPHS 


\title{
COMPLETE HOLOMORPHS
}

\author{
JoHN HARVEY
}

1. Introduction. Throughout this paper let $G$ be an additive group, and denote the group of all automorphisms of $G$ by $A(G)$ and the holomorph of $G$ by $K(G)$. Then $K(G)=A(G) \times G$, where $(\alpha, a)+(\beta, b)=$ $(\alpha \beta, a \beta+b)$ for all elements $(\alpha, a)$ and $(\beta, b)$ of $K(G)$. We prove that if $G$ is abelian and $x \rightarrow 2 x$ is an automorphism of $G$, then $K(G)$ is complete if and only if $G^{\prime}=1 \times G$ is a characteristic subgroup of $K(G)$. From this it follows that if $G$ is abelian, $x \rightarrow 2 x$ is an automorphism of $G$, and $A(G)$ is abelian, then $K(G)$ is complete.

In $\S 3$ we derive analogous results for ordered abelian groups. Then we show that any divisible, torsion free, abelian group can be ordered so that its o-holomorph is o-complete. It is known (see [2]) that the holomorph of a non-abelian group is not complete. In $\S 4$ we give an example of a non-abelian o-group with an o-complete o-holomorph. Finally, we show that the lexicographically ordered direct sum of two o-complete groups is again o-complete.

2. Complete holomorphs. Recall that a group is complete if it has a trivial center and all of its automorphisms are inner.

In 1957, W. Peremans [3] investigated under what conditions the holomorph of an abelian group is complete. He was able to derive a necessary and sufficient condition for the holomorph to be complete when $x \rightarrow 2 x$ is an automorphism of the group. Using this result he was then able to prove that if $x \rightarrow 2 x$ is an automorphism of the group and if the group is either directly indecomposable, the direct sum of cyclic groups, or is divisible, then the holomorph is complete.

We derive a necessary and sufficient condition which is simpler in statement than that of Peremans. However, before this theorem can be proved some preliminary lemmas are necessary which have independent interest. Let $B$ be a subgroup of $A(G)$, and let $\tau$ be a mapping from $B$ into $G$. Then $\tau$ is a crossed homomorphism if for all $\alpha$ and $\beta$ in $B$,

$$
(\alpha \beta) \tau=(\alpha \tau) \beta+\beta \tau
$$

Lemma 2.1. Let $G$ be an abelian group. If $\tau$ is a crossed homomorphism of $A(G)$ into $G$, then the mapping $\chi$ of $K(G)$ into itself defined by

Received September 2, 1960. This research was partially supported by a grant from the National Science Foundation, and represents a portion of a dissertation submitted to the Graduate School of Tulane University. The author wishes to express his appreciation to Professor P. F. Conrad for his help in preparing this paper. 


$$
(\alpha, \alpha) \chi=(\alpha, \alpha \tau+a)
$$

is an automorphism of $K(G)$ which induces the identity automorphism on $G^{\prime}$. Conversely, if $\chi$ is an automorphism of $K(G)$ and if $\chi$ induces the identity automorphism on $G^{\prime}$, then there exists a crossed homomorphism $\tau$ mapping $A(G)$ into $G$ such that for all $(\alpha, a)$ in $K(G)$

$$
(\alpha, a) \chi=(\alpha, \alpha \tau+a) .
$$

Proof. The first part of this lemma follows by an easy computation which we leave to the reader.

Suppose that $\chi$ is an element of the automorphism group of $K(G)$ and that $\chi$ induces the identity automorphism on $G^{\prime}$. If $(\alpha, a)$ is an element of $K(G)$, then

$$
\begin{aligned}
(\alpha, \alpha) \chi & =(\alpha, 0) \chi+(1, a) \chi \\
& =(\alpha \sigma, \alpha \tau)+(1, a) \\
& =(\alpha \sigma, \alpha \tau+a),
\end{aligned}
$$

where $1 \sigma=1$ and $1 \tau=0$.

For $(\alpha, \alpha)$ and $(\beta, b)$ belonging to $K(G)$ we have

$$
\begin{aligned}
((\alpha, a)+(\beta, b)) \chi & =(\alpha \beta, a \beta+b) \chi \\
& =((\alpha \beta) \sigma,(\alpha \beta) \tau+a \beta+b)
\end{aligned}
$$

and

$$
\begin{aligned}
(\alpha, a) \chi+(\beta, b) \chi & =(\alpha \sigma, \alpha \tau+a)+(\beta \sigma, \beta \tau+b) \\
& =(\alpha \sigma \beta \sigma,(\alpha \tau+a) \beta \sigma+\beta \tau+b)
\end{aligned}
$$

Therefore,

$$
(\alpha \beta) \tau+a \beta=(\alpha \tau)(\beta \sigma)+a(\beta \sigma)+\beta \tau .
$$

If $\alpha=1$, then for all $a$ in $G, a \beta=a(\beta \sigma)$. Hence, for all $\beta$ in $A(G)$, $\beta=\beta \sigma$, and thus, $\sigma=1$. Thus, we have that $(\alpha \beta) \tau=(\alpha \tau) \beta+\beta \tau$, and $(\alpha, \alpha) \chi=(\alpha, \alpha \tau+\alpha)$.

LEMмA 2.2. If $G$ is an abelian group such that $x \rightarrow 2 x$ is an automorphism of $G$, and if $\chi$ is an automorphism of $K(G)$ such that $G^{\prime} \chi=G^{\prime}$, then $\chi$ is an inner automorphism of $K(G)$.

Proof. Since $G^{\prime} \chi=G^{\prime}$, there exists an inner automorphism $\delta$ of $K(G)$ such that $\chi=\delta$ on $G^{\prime}$. Let $\chi_{1}=\chi \delta^{-1}$. Then $\chi_{1}$ induces the identity automorphism on $G^{\prime}$, and if we can show that $\chi_{1}$ is an inner automorphism of $K(G)$, then we will also have shown that $\chi$ is an inner automorphism of $K(G)$. Hence, we will consider $\chi_{1}$ instead of $\chi$. 
By Lemma 2.1 we know that $(\alpha, a) \chi_{1}=(\alpha, \alpha \tau+a)$ where $\tau$ is a crossed homomorphism mapping $A(G)$ into $G$. Let $\overline{2}$ be the automorphism $a \overline{2}=2 a$, where $a$ is in $G$. Since $\tau$ is a crossed homomorphism and $\alpha \overline{2}=\overline{2} \alpha$ for all $\alpha$ in $A(G)$, we have

$$
2(\alpha \tau)+\overline{2} \tau=(\alpha \overline{2}) \tau=(\overline{2} \alpha) \tau=\overline{(2} \tau) \alpha+\alpha \tau .
$$

Hence,

$$
\alpha \tau=(\overline{2} \tau) \alpha-\overline{2} \tau
$$

Then, for all $(\alpha, a)$ in $K(G)$,

$$
\begin{aligned}
(1, \overline{2} \tau)+(\alpha, a)-(1, \overline{2} \tau) & =(\alpha,(\overline{2} \tau) \alpha+a)+(1,-\overline{2} \tau) \\
& =(\alpha,(\overline{2} \tau) \alpha-\overline{2} \tau+a) \\
& =(\alpha, \alpha \tau+a) \\
& =(\alpha, a) \chi_{1} .
\end{aligned}
$$

Lemma 2.3. Suppose that $G$ is an abelian group and that $D$ is a non-trivial subgroup of $A(G)$. Then the natural splitting extension $H$ of $G$ by $D$ has a non-trivial center if and only if there exists a nonzero element $a$ of $G$ such that $a \alpha=a$ for all elements $\alpha$ of $D$.

Proof. We have that $H=D \times G$ where $(\alpha, a)+(\beta, b)=(\alpha \beta, a \beta+b)$ for all $(\alpha, a)$ and $(\beta, b)$ in $H$.

Suppose there exists a nonzero element $a$ of $G$ such that $a \alpha=a$ for all $\alpha$ in $D$. Then $(1, a)$ is an element of the center of $H$, and $(1, a) \neq$ $(1,0)$.

Now suppose that $(\beta, b)$ is an element of the center of $H$ such that $(\beta, b) \neq(1,0)$. Then, for all $(\alpha, a)$ in $H$,

$$
(\alpha, a)+(\beta, b)=(\beta, b)+(\alpha, a) .
$$

Thus, for all $\alpha$ in $D$ and all $a$ in $G, \alpha \beta=\beta \alpha$, and $a \beta+b=b \alpha+a$. If $\alpha=1$, then, for all $a$ in $G, a \beta=a$. Thus, $\beta=1$. Hence, $b=b \alpha$ for all $\alpha$ in $D$, and since $\beta=1, b$ must be nonzero for otherwise $(\beta, b)=$ $(1,0)$.

THeorem 2.1. If $G$ is an abelian group in which $x \rightarrow 2 x$ is an automorphism, then $K(G)$ is complete if and only if $G^{\prime}$ is characteristic in $K(G)$.

Proof. It follows from Lemma 2.3 that the center of $K(G)$ is trivial since $x \rightarrow 2 x$ leaves no point of $G$ fixed. If $K(G)$ is complete, then every automorphism of $K(G)$ is inner, and thus, since $G^{\prime}$ is normal in $K(G), G^{\prime}$ is characteristic. 
Next suppose that $\chi$ is an automorphism of $K(G)$ and that $G^{\prime}$ is characteristic in $K(G)$. Then $G^{\prime} \chi=G^{\prime}$, and hence, by Lemma $2.2, \chi$ is an inner automorphism of $K(G)$. Thus, $K(G)$ is complete.

If $G$ is finite, then the theorem gives the known result that the holomorph $K(G)$ of an abelian group of odd order is complete if and only if $G^{\prime}$ is characteristic in $K(G)$. In this case the mapping $x \rightarrow 2 x$ is clearly an automorphism of $G$.

CoRollary 2.1. If $G$ is an abelian group in which $x \rightarrow 2 x$ is an automorphism and if $A(G)$ is abelian, then $K(G)$ is complete.

Proof. It is well known that the commutator subgroup of a group is always a characteristic subgroup; hence, if we can show that $G^{\prime}$ is the commutator subgroup of $K(G)$, then by theorem $2.1, K(G)$ will be complete.

Since $K(G) / G^{\prime}$ is isomorphic to $A(G)$ and $A(G)$ is abelian, $G^{\prime}$ contains the commutator subgroup. Also, for any $(1, a)$ in $K(G)$ and any $b$ in $G$,

$$
-(1, a)-(\overline{2}, b)+(1, a)+(\overline{2}, b)=(1, a) .
$$

Thus, every element of $G^{\prime}$ is a commutator.

3. o-complete o-holomorphs. The ideas of completeness and the holomorph can be carried over into the theory of (linearly) ordered groups. An o-group is o-complete if its center is trivial and all of its o-automorphisms are inner. Suppose that $G$ is an o-group and that the group $A_{0}(G)$ of all o-automorphisms of $G$ can be ordered. We define the o-holomorph of $G$ to be the subgroup $K_{0}(G)=A_{0}(G) \times G$ of $K(G)$. Let $(\alpha, a)$ be positive if $\alpha$ is positive or if $\alpha=1$ and $a$ is positive in $G$. Then it is easy to verify that $K_{0}(G)$ is an o-group with respect to this definition and that $G^{\prime}$ is a normal convex subgroup of $K_{0}(G)$.

It is known that an o-group is o-complete if and only if it is a direct summand in any o-group which contains it as a normal convex subgroup. The proof is a slight variation of the classical proof for non-ordered complete groups.

THEOREM 3.1. Let $G$ be an abelian o-group for which $A_{0}(G)$ can be ordered. If $\tau$ is a crossed homomorphism of $A_{0}(G)$ into $G$, then the mapping $\chi$ from $K_{0}(G)$ into $K_{0}(G)$ defined by

$$
(\alpha, a) \chi=(\alpha, \alpha \tau+a)
$$

is an order preserving automorphism of $K_{0}(G)$ which induces the identity automorphism on $G^{\prime}$. Conversely, if $\chi$ is an order preserving automorphism of $K_{0}(G)$ and if $\chi$ induces the identity automorphism on $G^{\prime}$, 
then there exists a crossed homomorphism $\tau$ mapping $A_{0}(G)$ into $G$ such that for all $(\alpha, a)$ in $K_{0}(G)$,

$$
(\alpha, a) \chi=(\alpha, \alpha \tau+\alpha) .
$$

The proof is identical with the proof of Lemma 2.1. One only need verify that the mapping $(\alpha, a) \chi=(\alpha, \alpha \tau+a)$ preserves order (when $(\alpha, a)$ is in $K_{0}(G)$ and $\tau$ is a crossed homomorphism). But if $1<\alpha$, then $(\alpha, \alpha)$ and $(\alpha, \alpha \tau+a)$ are positive, and if $\alpha=1$ and $0<a$, then $(\alpha, \alpha \tau+a)=$ $(1, a)$ is positive.

Corollary 3.1. Suppose that $G$ is an abelian o-group in which $x \rightarrow 2 x$ is an automorphism and for which $A_{0}(G)$ can be ordered. If $\chi$ is an order preserving automorphism of $K_{0}(G)$ such that $G^{\prime} \chi=G^{\prime}$, then $\chi$ is an inner automorphism of $K_{0}(G)$.

This corollary follows at once from the proof of Lemma 2.2 and the fact that an inner automorphism of an o-group is an o-automorphism.

If $G$ is an o-group, then a subgroup $C$ of $G$ is said to be o-characteristic if $C \delta=C$ for all $\delta$ in $A_{0}(G)$.

THEOREM 3.2. Suppose that $G$ is an abelian o-group in which $x \rightarrow 2 x$ is an automorphism and for which $A_{0}(G)$ can be ordered. Then $K_{0}(G)$ is o-complete if and only if $G^{\prime}$ is o-characteristic in $K_{0}(G)$.

The proof of this theorem is analogous to the proof of Theorem 2.1.

Suppose that $G$ is an o-group and that $C$ and $C^{\prime}$ are two convex subgroups of $G$. Then $C$ covers $C^{\prime}$ if $C$ contains $C^{\prime}$ and there is no convex subgroup of $G$ between $C$ and $C^{\prime}$. Let $\Gamma$ be the set of all ordered pairs $\left(G^{\alpha}, G_{\alpha}\right)$ of convex subgroups such that $G^{\alpha}$ covers $G_{\alpha}$. Define $\left(G^{\alpha}, G_{\alpha}\right)>\left(G^{\beta}, G_{\beta}\right)$ if $G_{\alpha}$ contains $G^{\beta}$. This orders $\Gamma$. We can regard $\Gamma$ as an ordered set $\alpha, \beta, \gamma, \cdots$. The order type of $\Gamma$ is the rank of $G$. The set $\Gamma$ will be called the rank set of $G$. The groups $G^{\alpha} / G_{\alpha}$ for $\alpha$ in $\Gamma$ are the components of $G$.

CoRollary 3.2. If $G$ is an abelian o-group in which $x \rightarrow 2 x$ is an automorphism and for which $A_{0}(G)$ can be ordered, and if $G$ has wellordered rank, than $K_{0}(G)$ is o-complete.

Before we prove this corollary, we shall prove a lemma concerning well-ordered subsets of an ordered set.

Lemma 3.1. If $L$ is an ordered set, if $W$ is a well-ordered convex subset of $L$, and if $f$ is a one-to-one, order preserving mapping of $L$ onto itself such that $f(\delta)=\delta$ where $\delta$ is the least element of $W$, then $f(\alpha)=\alpha$ for all $\alpha$ in $W$. 
Proof. Suppose $\alpha$ is any element of $W$ such that $\alpha \neq \delta$. Then $[\delta, \alpha]$ is a well-ordered subset of $L$. Suppose $f(\alpha) \neq \alpha$. Then either $f(\alpha)<\alpha$ or $f^{-1}(\alpha)<\alpha$. Without loss of generality we may assume that $f(\alpha)<\alpha$. Then $f$ is a one-to-one mapping of $[\delta, \alpha]$ into itself. Hence, $\alpha \leqq f(\alpha)$ which is a contradiction to our assumption. Thus, $f(\alpha)=\alpha$ for all $\alpha$ in $W$.

Proof of Corollary 2.2. The rank set of $K_{0}(G)$ is an ordered set. Since $G$ has well-ordered rank, the rank set of $K_{0}(G)$ contains a wellordered convex subset-the rank set of $G$. Now any order preserving automorphism of $K_{0}(G)$ induces a one-to-one, order preserving mapping of the rank set of $K_{0}(G)$ onto itself. By Lemma 3.1 this order preserving mapping is the identity on the rank set of $G$. But this means that $G^{\prime}$ is o-characteristic, and therefore by Theorem 3.2, we see that $K_{0}(G)$ is o-complete.

It is well known that a torsion free abelian group can be ordered, and as mentioned before, Peremans has shown that the holomorph of a divisible abelian group is complete. It does not seem likely that for every ordering of a divisible, torsion free, abelian group it will be possible to order the resulting group of order preserving automorphisms. However, Conrad [1] has proved the following useful result:

If $G$ is an o-group of well-ordered rank each of whose components is isomorphic to the additive group of rational numbers, then $A_{0}(G)$ can be ordered.

This result together with Corollary 3.2 gives us the following theorem.

THEOREm 3.3. Any divisible, torsion free, abelian group can be ordered so that

(1) $A_{0}(G)$ can be ordered and

(2) $K_{0}(G)$ is o-complete.

Proof. A divisible, torsion free, abelian group $G$ is a rational vector space. Hence we can choose a basis $A$ for $G$ and well-order $A$. If $g$ is any nonzero element of $G$, then $g=r_{1} \alpha_{1}+r_{2} \alpha_{2}+\cdots+r_{n} \alpha_{n}$ where the $r_{i}$ are nonzero rational numbers and the $\alpha_{i}$ are elements of the basis $A$. Without loss of generality we may assume that $\alpha_{1}<\alpha_{2}<\cdots<\alpha_{n}$ in the well-ordering of $A$. We will say that $g$ in $G$ is positive if $0<r_{n}$. Then $G$ is an o-group with well-ordered rank each of whose components is o-isomorphic to the rational numbers. Thus, by the result of Conrad stated above, $A_{0}(G)$ can be ordered, and by Corollary $3.2, K_{0}(G)$ is o-complete.

REMARK. It is well known that any torsion free abelian group is contained in a unique (to within an isomorphism) minimal divisible group 
which is also torsion free and abelian. Thus, any torsion free abelian group is contained is an o-complete group.

4. Examples of o-complete groups. This section will consist of several examples of o-complete groups and a theorem which concerns direct sums of o-complete groups.

A small amount of notation needs to be introduced at this time. If $G$ and $H$ are groups, then $\operatorname{Hom}(G, H)$ will denote the set of all homomorphisms mapping $G$ into $H$. Throughout the examples $\boldsymbol{R}$ will denote the additive group of real numbers with their natural order, $R$ will denote the additive group of rational numbers with their (unique) natural order, $\boldsymbol{R}^{\prime}$ will denote the multiplicative group of positive real numbers, and $R^{\prime}$ will denote the multiplicative group of positive rational numbers.

EXAmple I. The o-automorphism group of $\boldsymbol{R}$ is (isomorphic to) $\boldsymbol{R}^{\prime}$. Give $\boldsymbol{R}^{\prime}$ its natural order. Then $K_{0}(\boldsymbol{R})$ is o-complete by Corollary 3.2. It should be noted that $K_{0}(\boldsymbol{R})$ is (isomorphic to) the multiplicative group of $2 \times 2$ matrices of the form

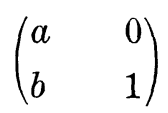

where $a$ is in $\boldsymbol{R}^{\prime}$ and $b$ is in $\boldsymbol{R}$. Such a matrix is posivive if $1<a$ or $1=a$ and $0<b$. Also note that $K_{0}(\boldsymbol{R})$ is of rank two.

EXAMPLE II. Let $M$ be the additive group of all rationals of the form $m / 2^{n}$ where $m$ and $n$ are integers, and let $M$ have its natural order. Let $N$ be the cyclic subgroup of $R^{\prime}$ generated by 2 . Notice that neither $M$ nor any of its proper subgroups are divisible; hence $\operatorname{Hom}(R, M)=0$.

Let $G=R \oplus M$ where $\left(a_{1}, a_{2}\right)$ in $G$ is positive if $a_{1}>0$ or $a_{1}=0$ and $a_{2}>0$. Then $G$ is an abelian o-group of rank 2 . Then since $\operatorname{Hom}(R, M)=0$, if $\phi$ is an element of $A_{0}(G)$ then $\phi=\left(p_{1}, p_{2}\right)$ where $p_{1}$ is in $R^{\prime}$ and $p_{2}$ is in $N$, and conversely, if $\phi=\left(p_{1}, p_{2}\right)$ where $p_{1}$ is in $R^{\prime}$ and $p_{2}$ is in $N$, then $\phi$ is in $A_{0}(G)$, i.e., $A_{0}(G)=R^{\prime} \otimes N$. Now $R^{\prime}$ is a free abelian group of countable rank, and so is $R^{\prime} \otimes N$. Thus, $R^{\prime}$ is isomorphic to $R^{\prime} \otimes N$. Define an element of $R^{\prime} \otimes N$ to be positive if its image in $R^{\prime}$ is positive, where $R^{\prime}$ is given its natural order. Then $R^{\prime} \otimes N$ is an abelian o-group of rank one, and so by Corollary $3.2 K_{0}(G)$ is o-complete and of rank three.

Example III. Let $G=R \oplus R$ where $\left(a_{1}, a_{2}\right)$ in $G$ is positive if $0<a_{1}$ or $0=a_{1}$ and $0<a_{2}$. Then it is easy to show that $A_{0}(G)$ is isomorphic to the group of $2 \times 2$ matrices of the form 


$$
\left(\begin{array}{ll}
a & c \\
0 & b
\end{array}\right)
$$

where $a$ and $b$ are elements of $R^{\prime}$ and $c$ is in $R$. Such a matrix is positive if $1<a$ or $1=a$ and $1<b$ or $1=a=b$ and $0<c$. Then, $A_{0}(G)$ is an o-group of rank three. By Corollary 3.2, $K_{0}(G)$ is o-complete, and we observe that $K_{0}(G)$ is of rank five.

The above three examples show that there are o-complete groups of rank two, three, and five. Using the next theorem we can show that there are o-complete groups for every finite rank greater than one.

Throughout the following discussion, let $D$ and $N$ be o-groups. To avoid confusion let the identity element of $D$ be denoted by $\theta$ and that of $N$ by 0 . Whenever $G=D \oplus N$ we will always order $G$ as follows: $(\alpha, a)$ in $G$ is positive if $\theta<\alpha$ or $\theta=\alpha$ and $0<a$.

LEMma 4.1. Suppose that $G=D \oplus N$ and that the center of $N$ is trivial. If $N^{\prime}=\theta \times N$ is o-characteristic in $G$, then $A_{0}(G)$ is isomorphic to $A_{0}(D) \otimes A_{0}(N)$.

Proof. If $\phi$ is in $A_{0}(G)$ and if $(\alpha, a)$ and $(\beta, b)$ are in $G$, then

$$
\begin{aligned}
(\alpha, a) \phi & =(\alpha, 0) \phi+(\theta, a) \phi=(g(\alpha), h(\alpha))+(\theta, P(a)) \\
& =(g(\alpha), h(\alpha)+P(a))
\end{aligned}
$$

where $P$ is in $A_{0}(N)$ and $h(\theta)=0$.

$$
\begin{aligned}
((\alpha, a)+(\beta, b)) \phi & =(\alpha+\beta, a+b) \phi \\
& =(g(\alpha+\beta), h(\alpha+\beta)+P(a+b)) \\
(\alpha, a) \phi+(\beta, b) \phi & =((g(\alpha), h(\alpha)+P(\alpha))+(g(\beta), h(\beta)+P(b)) \\
& =(g(\alpha)+g(\beta), h(\alpha)+P(a)+h(\beta)+P(b)) .
\end{aligned}
$$

Hence, $g(\alpha+\beta)=g(\alpha)+g(\beta)$, and it follows by an easy argument that $g$ is an element of $A_{0}(D)$. Also,

$$
h(\alpha+\beta)+P(\alpha)=h(\alpha)+P(a)+h(\beta) .
$$

If $\alpha=\theta$, then for all $\alpha$ in $N$ and $\beta$ in $D$,

$$
h(\beta)+P(a)=P(a)+h(\beta) .
$$

Therefore, $h(\beta)$ is in the center of $N$ (which is trivial) for all $\beta$ in $D$, and hence, $(\alpha, a) \phi=(g(\alpha), P(\alpha))$. It follows that the mapping of $\phi$ upon $(g, P)$ is an isomorphism of $A_{0}(G)$ onto $A_{0}(D) \otimes A_{0}(N)$.

Theorem 4.1. Suppose that $G=D \oplus N$. Then $G$ is o-complete if and only if $D$ and $N$ are o-complete and $N^{\prime}$ is o-characteristic in $G$. 


\section{Proof. Let us denote the center of a group $G$ by $Z(G)$.}

First suppose that $G$ is o-complete. Then since $0=Z(G)=Z(D) \oplus Z(N)$, $D$ and $N$ have trivial centers. Consider $\delta$ in $A_{0}(N)$ and $(\alpha, \alpha)$ in $G$. Define the mapping $\phi$ of $G$ into itself by

$$
(\alpha, \alpha) \phi=(\alpha, a \delta) \text {. }
$$

Clearly, $\phi$ is in $A_{0}(G)$, and since $G$ is o-complete there exists $(\beta, b)$ in $G$ such that

$$
\begin{aligned}
(\alpha, a \delta) & =(\alpha, a) \phi=-(\beta, b)+(\alpha, a)+(\beta, b) \\
& =(-\beta+\alpha+\beta,-b+a+b) .
\end{aligned}
$$

Thus, $a \delta=-b+a+b$, and hence, $N$ is o-complete. By a similar argument $D$ is o-complete. Since $G$ is o-complete and $N^{\prime}$ is a normal convex subgroup of $G$, it is clear that $N^{\prime}$ is o-characteristic in $G$.

Finally, suppose that $D$ and $N$ are o-complete and that $N^{\prime}$ is o-characteristic in $G$. If $\phi$ is in $A_{0}(G)$, then by Lemma 4.1 , we have that $\phi$ is equivalent to $(g, P)$, where $g$ is in $A_{0}(D)$ and $P$ is in $A_{0}(N)$. Since $D$ and $N$ are both o-complete there exists $\beta$ in $D$ and $b$ in $N$ such that for all $a$ in $N, P(a)=-b+a+b$, and for all $\alpha$ in $D, g(\alpha)=-\beta+$ $\alpha+\beta$. Therefore, for all $(\alpha, a)$ in $G$.

$$
\begin{aligned}
(\alpha, a) \phi & =(-\beta+\alpha+\beta,-b+a+b) \\
& =(-\beta,-b)+(\alpha, a)+(\beta, b) \\
& =-(\beta, b)+(\alpha, \alpha)+(\beta, b) .
\end{aligned}
$$

Thus, $\phi$ is an inner automorphism. Since $Z(D)$ and $Z(N)$ are both trivial it is clear that $Z(G)$ must be trivial, and hence, $G$ is o-complete.

The second half of Theorem 4.1 may be used to construct further examples of o-complete groups. Using the examples given in the first portion of this section we see that we can easily construct o-complete groups for any finite rank greater than one.

Suppose that $G$ is an o-group such that $A_{0}(G)$ can be ordered and $K_{0}(G)$ is o-complete. Then $A_{0}\left(K_{0}(G)\right)$ is isomorphic to $K_{0}(G)$, and hence, inherits an order. Since $K_{0}(G)$ is o-complete and since every o-complete group is a direct summand of any o-group which contains it as a normal convex subgroup, we have that $K_{0}\left(K_{0}(G)\right)=T \oplus K_{0}(G)$ where $T$ is o-isomorphic to $A_{0}\left(K_{0}(G)\right)$. Therefore, $K_{0}\left(K_{0}(G)\right)$ is o-complete if and only if $K_{0}(G)$ is o-characteristic (by Theorem 4.1). In particular, if $K_{0}(G)$ has well-ordered rank, then $K_{0}\left(K_{0}(G)\right)$ is o-complete. Thus, the second o-holomorphs of any one of the examples are o-complete.

Added in Proof. It has been pointed out to the author by Professor W. Peremans that Theorem 2.1 of this paper has previously appeared 
as "Satz*" on page 101 of W. Specht, Gruppentheorie (Springer, 1956). However the proof given by Specht is different from the one given here, and the proof given by Specht is not applicable for o-groups (c.f. Theorem 3.2)

\section{BIBLIOGRAPHY}

1. P. Conrad, A correction and improvement of a theorem on ordered groups, Proc. Amer. Math. Soc., 10 (1959), 182-184.

2. W. H. Mills, On the non-isomorphism of certain holomorphs, Trans. Amer. Math. Soc., 74 (1953), 428-443.

3. W. Peremans, Completeness of holomorphs, Indag. Math., 19 (1957), 607-619.

TUlane UNIVERSITY 


\title{
PACIFIC JOURNAL OF MATHEMATICS
}

\section{EDITORS}

\author{
Ralph S. Phillips \\ Stanford University \\ Stanford, California \\ F. H. BrownelL \\ University of Washington \\ Seattle 5 , Washington
}

A. L. Whiteman

University of Southern California

Los Angeles 7. California

L. J. PAIGE

University of California

Los Angeles 24, California

\section{ASSOCIATE EDITORS}

\author{
E. F. BECKENBACH \\ T. M. CHERRY
} \\ D. DERRY \\ M. OHTSUKA \\ UNIVERSITY OF BRITISH COLUMBIA \\ CALIFORNIA INSTITUTE OF TECHNOLOGY \\ UNIVERSITY OF CALIFORNIA \\ MONTANA STATE UNIVERSITY \\ UNIVERSITY OF NEVADA \\ NEW MEXICO STATE UNIVERSITY \\ OREGON STATE COLLEGE \\ UNIVERSITY OF OREGON \\ OSAKA UNIVERSITY \\ UNIVERSITY OF SOUTHERN CALIFORNIA
}

H. L. ROYDEN

E. SPANIER
E. G. STRAUS

F. WOLF

\section{SUPPORTING INSTITUTIONS}

\author{
STANFORD UNIVERSITY \\ UNIVERSITY OF TOKYO \\ UNIVERSITY OF UTAH \\ WASHINGTON STATE COLLEGE \\ UNIVERSITY OF WASHINGTON \\ AMERICAN MATHEMATICAL SOCIETY \\ CALIFORNIA RESEARCH CORPORATION \\ HUGHES AIRCRAFT COMPANY \\ SPACE TECHNOLOGY LABORATORIES \\ NAVAL ORDNANCE TEST STATION
}

Mathematical papers intended for publication in the Pacific Journal of Mathematics should be typewritten (double spaced), and the author should keep a complete copy. Manuscripts may be sent to any one of the four editors. All other communications to the editors should be addressed to the managing editor, L. J. Paige at the University of California, Los Angeles 24, California.

50 reprints per author of each article are furnished free of charge; additional copies may be obtained at cost in multiples of 50 .

The Pacific Journal of Mathematics is published quarterly, in March, June, September, and December. The price per volume (4 numbers) is $\$ 12.00$; single issues, $\$ 3.50$. Back numbers are available. Special price to individual faculty members of supporting institutions and to individual members of the American Mathematical Society: $\$ 4.00$ per volume; single issues, $\$ 1.25$.

Subscriptions, orders for back numbers, and changes of address should be sent to Pacific Journal of Mathematics, 103 Highland Boulevard, Berkeley 8, California.

Printed at Kokusai Bunken Insatsusha (International Academic Printing Co., Ltd.), No. 6 , 2-chome, Fujimi-cho, Chiyoda-ku, Tokyo, Japan.

PUBLISHED BY PACIFIC JOURNAL OF MATHEMATICS, A NON-PROFIT CORPORATION

The Supporting Institutions listed above contribute to the cost of publication of this Journal, but they are not owners or publishers and have no responsibility for its content or policies. 


\section{Pacific Journal of Mathematics}

\section{Vol. 11, No. $3 \quad$ BadMonth, 1961}

Errett Albert Bishop, A generalization of the Stone-Weierstrass theorem ..........

Hugh D. Brunk, Best fit to a random variable by a random variable measurable with

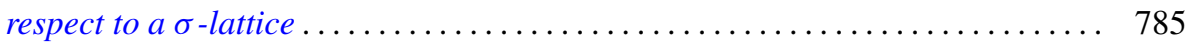

D. S. Carter, Existence of a class of steady plane gravity flows . . . . . . . . . . 803

Frank Sydney Cater, On the theory of spatial invariants ............... 821

S. Chowla, Marguerite Elizabeth Dunton and Donald John Lewis, Linear

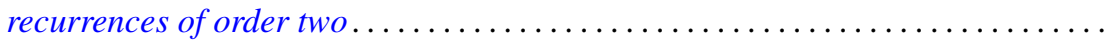

Paul Civin and Bertram Yood, The second conjugate space of a Banach algebra as

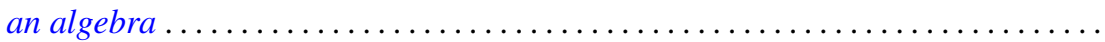

William J. Coles, Wirtinger-type integral inequalities .

Shaul Foguel, Strongly continuous Markov processes ....................

David James Foulis, Conditions for the modularity of an orthomodular lattice ...... Jerzy Górski, The Sochocki-Plemelj formula for the functions of two complex variables.

John Walker Gray, Extensions of sheaves of associative algebras by non-trivial

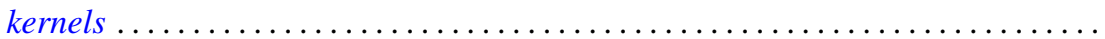

Maurice Hanan, Oscillation criteria for third-order linear differential equations .... 919 Haim Hanani and Marian Reichaw-Reichbach, Some characterizations of a class of unavoidable compact sets in the game of Banach and Mazur .............

John Grover Harvey, III, Complete holomorphs . . . . . . . . . . . . . . . . 961 Joseph Hersch, Physical interpretation and strengthing of M. Protter's method for vibrating nonhomogeneous membranes; its analogue for Schrödinger's

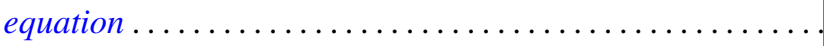

James Grady Horne, Jr., Real commutative semigroups on the plane...

Nai-Chao Hsu, The group of automorphisms of the holomorph of a group...

F. Burton Jones, The cyclic connectivity of plane continua

John Arnold Kalman, Continuity and convexity of projections and barycentric

coordinates in convex polyhedra

Samuel Karlin, Frank Proschan and Richard Eugene Barlow, Moment inequalities of

Pólya frequency functions .

Tilla Weinstein, Imbedding compact Riemann surfaces in 3-space. .

Azriel Lévy and Robert Lawson Vaught, Principles of partial reflection in the set

theories of Zermelo and Ackermann

Donald John Lewis, Two classes of Diophantine equations .....

Daniel C. Lewis, Reversible transformations . . .

Gerald Otis Losey and Hans Schneider, Group membership in rings and

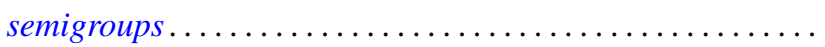

M. N. Mikhail and M. Nassif, On the difference and sum of basic sets of

polynomials.

Alex I. Rosenberg and Daniel Zelinsky, Automorphisms of separable algebras .

Robert Steinberg, Automorphisms of classical Lie algebras .... 